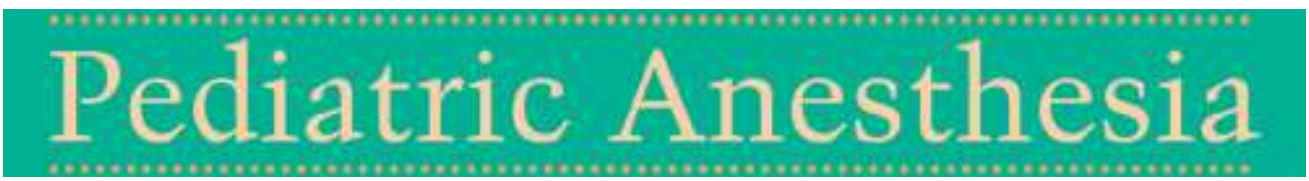

\title{
Upper airway size and configuration during propofol-based sedation for magnetic resonance imaging: an analysis of 138 infants and children
}

\begin{tabular}{|c|c|}
\hline Journal: & Pediatric Anesthesia \\
\hline Manuscript ID: & PAN-2010-0327.R2 \\
\hline Manuscript Type: & Original Paper \\
\hline $\begin{array}{r}\text { Date Submitted by the } \\
\text { Author: }\end{array}$ & 04-Aug-2010 \\
\hline Complete List of Authors: & $\begin{array}{l}\text { Machata, Anette-Marie; Medical University of Vienna, Department of } \\
\text { Anaesthesia, General Intensive Care and Pain Therapy } \\
\text { Kabon, Barbara; Medical University of Vienna, Department of } \\
\text { Anaesthesia, General Intensive Care and Pain Therapy } \\
\text { Willschke, Harald; Medical University of Vienna, Department of } \\
\text { Anaesthesia, General Intensive Care and Pain Therapy } \\
\text { Prayer, Daniela; Medical University of Vienna, Department of } \\
\text { Radiology } \\
\text { Marhofer, Peter; Medical University of Vienna, Department of } \\
\text { Anaesthesia, General Intensive Care and Pain Therapy }\end{array}$ \\
\hline Key Words: & child $<$ Airway, child $<$ Age, infant $<$ Age \\
\hline
\end{tabular}


Upper airway size and configuration during propofol-based sedation

for magnetic resonance imaging: an analysis of 138 infants and

children

\author{
ANETTE-MARIE MACHATA ${ }^{1}{ }^{1}$, BARBARA KABON ${ }^{1}{ }^{1}$, HARALD WILLSCHKE \\ MD ${ }^{1}$, DANIELA PRAYER MD ${ }^{2}$ AND PETER MARHOFER MD ${ }^{1}$ \\ ${ }^{1}$ Department of Anesthesia, General Intensive Care and Pain Therapy, \\ ${ }^{2}$ Department of Radiology, \\ Medical University of Vienna, Austria
}

Correspondence to:

Anette-Marie Machata

Department of Anesthesia, General Intensive Care and Pain Therapy

Medical University of Vienna, General Hospital

Waehringer Guertel 18-20

A-1090 Vienna

Austria, Europe

anette-marie.machata@meduniwien.ac.at

Running title: Pediatric upper airway measured with MRI 


\begin{abstract}
Summary
Background

Propofol is widely used for pediatric sedation. However, increasing depth of propofol sedation is associated with airway narrowing and obstruction. The aim of this study was to objectively assess airway patency during a low-dose propofol-based sedation regimen by measuring upper airway size and configuration with MRI in spontaneously breathing infants and children.
\end{abstract}

\title{
Methods
}

Magnetic resonance images of the upper airway were obtained in 138 infants and children, aged up to 6 years. Cross-sectional area, anteroposterior dimension, and transverse dimension were measured at the level of the soft palate, the base of the tongue, and the tip of the epiglottis. Sedation was induced with i.v. midazolam $0.1 \mathrm{mg} \cdot \mathrm{kg}^{-1}$, nalbuphine $0.1 \mathrm{mg} \cdot \mathrm{kg}^{-1}$ and propofol $1 \mathrm{mg} \cdot \mathrm{kg}^{-1}$, and maintained with propofol $5 \mathrm{mg} \cdot \mathrm{kg}^{-1} \cdot \mathrm{h}^{-1}$.

\section{Results}

Median (IQR) age was $36(15,48)$ months, and mean body weight was $13,7 \pm 5,6 \mathrm{~kg}$. Airway patency was maintained in all infants and children. The narrowest part of the pharyngeal airway was measured at the level of the base of the tongue. Anteroposterior dimensions were narrower than transverse dimensions in all age groups at all measurement sites. Transverse dimensions increased with age at all measurement sites, while anteroposterior dimensions did not increase comparably. No patient demonstrated respiratory or cardiovascular adverse events. All MRI were completed successfully without sedation failure.

\section{Conclusion}

Airway patency was maintained in all infants and children sedated with this low-dose propofol-based sedation regimen.

Keywords: Anesthesia, pediatric; Anesthetic techniques, i.v.; Anesthetics i.v., propofol; Airway, anatomy; Brain, magnetic resonance imaging; 
Infants and children require deep sedation for successful management of magnetic resonance imaging (MRI) examinations. The noisy environment in the MRI suite and the duration of the examinations preclude a lower sedation state in these patients. Propofol is widely used for pediatric sedation, but increasing depth of propofol anesthesia is associated with the risk of upper airway obstruction $(1,2)$. Because maintenance of a patent airway during MRI procedures under spontaneous respiration is of utmost importance, airway management is still controversial in this setting. Sedation in spontaneously breathing children is advocated by several authors (3-5), others prefer laryngeal mask airway insertion (6) or tracheal intubation to secure the airway $(7,8)$. An appropriate low propofol dosage which maintains spontaneous respiration and ensures sufficient sedation for successful MRI completion would probably minimize the risk of airway obstruction.

In a previous study we described the safety and efficacy of a propofol-based sedation regimen for MRI examinations in 500 infants and children (9). Airway patency was only deduced from normal peripheral oxygen saturation and normal end-tidal carbon dioxide values in these patients. However, MRI allows direct determination of upper airway size and configuration, even if these measurements were only performed in a small number of pediatric patients until now (10-12).

Therefore, the aim of this study was to objectively assess airway patency by measuring upper airway size and configuration with MRI in spontaneously breathing infants and children sedated with the aforementioned propofol-based regimen. 


\section{Materials and Methods}

\section{Patients}

With ethics hospital committee approval, 150 consecutive ASA I and II infants and children, aged up to 6 years, who required sedation for elective cranial MRI examination, were enrolled in this study. Informed consent was obtained from the parents of all infants and children.

Exclusion criteria were ASA status $\geq$ III, severe pulmonary or cardiovascular disease, anatomic airway abnormalities or extreme tonsillar hypertrophy, gastroesophageal reflux, a body weight of at least $30 \%$ more than ideal, a history of propofol intolerance, or known fat metabolism disorder.

\section{Procedure}

On the day of the procedure, all patients were admitted with their parents at the pediatric day care ward. An intravenous access was obtained, and then the patient was transferred to the MRI suite accompanied by his/her parents. In a preprocedure interview, all parents were instructed about patient fasting as recommended by the "American Society of Anesthesiologists Preprocedure Fasting guidelines" (13,14).

After the patients received midazolam $0.1 \mathrm{mg} \cdot \mathrm{kg}^{-1}$ intravenously, they were moved into the MRI suite.

Then, nalbuphine $0.1 \mathrm{mg} \cdot \mathrm{kg}^{-1}$, followed by a loading dose of propofol $1 \mathrm{mg} \cdot \mathrm{kg}^{-1}$ was administered. Supplemental doses of propofol $0.5 \mathrm{mg} \cdot \mathrm{kg}^{-1}$ were administered until adequate sedation was achieved. Sedation was considered adequate, when the patient slept, arousable only with significant physical stimulation (University of Michigan Sedation Scale (UMSS3)) (15). The maintenance of spontaneous respiration was verified.

All children wear earphones to diminish the stimulation caused by the noise during MRI acquisition phases. 
The head position was standardized in a neutral position such that the angle between the horizontal plane of the MRI table and a line connecting the tragus of the ear and the lateral corner of the eye was $110^{\circ}(10,11)$. No devices (nasal or oral airway) that assist with patency of the airway were used, and no attempt was made to open or close the mouth (12). Furthermore, no attempt was made to obtain images at a certain timepoint of the respiratory cycle.

Supplemental oxygen was provided by pediatric face mask with a gas flow rate of $21 \cdot \mathrm{min}^{-1}$, and sedation was maintained with propofol $5 \mathrm{mg} \cdot \mathrm{kg}^{-1} \cdot \mathrm{h}^{-1}$ using syring pumps suitable for MRI.

Special monitors, not interfering with the MRI procedure, were placed in the examination room and in the observation room to monitor vital signs. Heart rate, arterial oxygen saturation $\left(\mathrm{SpO}_{2}\right)$ and end-tidal carbon dioxide $\left(\mathrm{EtCO}_{2}\right)$, sampled via special nasal cannula, were monitored continuously during the procedure and recorded at 5 min intervals by the anesthesiologist. Non-invasive arterial pressure was determined immediately before induction of sedation and at the end of the examination, but not during the MRI procedure, as stimulation from the blood pressure cuff would arouse an appropriately sedated child (14). Intervention was considered necessary when a decrease in $\mathrm{SpO}_{2}$ to $<94 \%$, an increase in $\mathrm{EtCO}_{2}>50 \mathrm{mmHg}$, apnea (cessation of spontaneous respiration for 20 seconds), bradycardia ( $>20 \%$ decrease in heart rate from baseline), or arrhythmia occurred.

For safety reasons, a camera was placed in the MRI suite to monitor patients, and the anesthesiologist closely observed the patients while sedated and provided interventions as needed: In case of inadequate sedation, such as spontaneous movements and agitation during the imaging process, an additional bolus of propofol $0.5 \mathrm{mg} \cdot \mathrm{kg}^{-1}$ was administered, and the propofol infusion rate was increased by $1 \mathrm{mg} \cdot \mathrm{kg}^{-1} \cdot \mathrm{h}^{-1}$ up to a total of $8 \mathrm{mg} \cdot \mathrm{kg}^{-1} \cdot \mathrm{h}^{-1}$. In case of coughing or suspected airway obstruction, the MRI examination was interrupted, the patient was taken out of the MRI unit, and the airway patency was assessed. When partial airway 
occlusion was diagnosed, the neck was again extended slightly and the chin was supported with a tape. When total airway occlusion was noted, bag-valve-mask ventilation was initiated, and a laryngeal mask airway was inserted, if necessary. Tracheal intubation was performed, when these maneuvers did not relieve obstruction.

After the MRI examination was completed, the propofol infusion was terminated, and the patients were transferred from the MRI suite to the induction room.

\section{Measurements}

Magnetic resonance images were obtained using a 1.5T Philips Achieva Release 1.2.2 scanner (Philips, Medizinische Systeme GmbH, Austria) with a master/NOVA gradient strength and a circular polarized birdcage headcoil. A M2D FFE sequence slice thickness $10 \mathrm{~mm} / 10 \mathrm{~mm}$ gap was used as a scout view in a three-plane gradient echo. Then a T1 3D FFE axial/oblique sequence was used. This sequence allows unequivocal recognition of the landmarks that were used for measurements. After the examinations, DICOM images were stored on computer and analyzed by a single investigator blinded to patient data. After image magnification (x 3), anteroposterior dimension, transverse dimension, and cross-sectional area (CSA) were determined at the level of the soft palate, the base of the tongue, and the tip of the epiglottis using image analysis software (Osirix Imaging Software, version 3.6.1) (figures 1-3). Because image acquisition at different phases of the respiratory circle was impossible in this setting, measurements were obtained from two subsequent slices at each anatomic level, and the average of both was calculated.

\section{Data analysis}

Data were descriptively analyzed and presented as median (IQR) or mean (SD) depending on their distribution. Normal distribution was assessed with q-q plot and Shapiro-Wilk test.

CSA measurements among the three age groups were compared with Kruskal-Wallis test. Friedman test was used to compare paired nonparametric data (differences between pharyngeal dimensions at different levels). Post hoc pairwise comparisons were performed 
with Wilcoxon rank sum test. To compensate for multiple comparisons the Bonferroni correction was used. Pearson's $r$ was used to determine the relationship between CSA and age. A $P<0.05$ was considered statistically significant. SPSS software was used for all analyses. 


\section{Results}

One hundred fifty patients were initially enrolled in the study. Twelve were excluded because of various reasons that included abnormal laryngeal anatomy (4), technical poor images (2), and data retrieval problems (6). From the remaining 138 patients, 60 were boys and 78 were girls. Median (IQR) age was $36(15,48)$ months; mean body weight was $13,7 \pm 5,6 \mathrm{~kg}$, median height was $95(78,104) \mathrm{cm}$. Patients were divided in three age groups: infants $<12$ months, children between 12 and $<36$ months, and $>36$ months. Pertinent data are shown in table 1 .

Airway patency was maintained in all patients at the 3 measurement sites (table 1). The narrowest part of the pharyngeal airway was measured at the level of the base of the tongue in all patients. Anteroposterior dimensions, transverse dimensions, and also CSA were significantly smaller at the level of the base of the tongue compared to the soft palate and epiglottis level, which were similar. Anteroposterior dimensions were narrower than transverse dimensions in all age groups at all measurement sites (table 1).

Transverse dimensions increased with age at all measurement sites, while anteroposterior dimensions did not increase comparably. There was a significant linear increase of CSA with age at all levels. However, the interindividual variation was wide, and the correlation was only moderate (figure 4).

During either phase of the study, no patient demonstrated clinically significant upper airway obstruction as manifested by decreased oxygen saturation or increased capnometric values. None of our patients suffered from cardiovascular events such as bradycardia or arrhythmia during and after sedation. Paradoxical reaction to sedation or agitation was not seen in our patients. All scheduled MRI examinations could completed without sedation failure. 


\section{Discussion}

To our knowledge, this prospective, observational study documents the largest case series of MRI measurements of the pediatric airway in the recent literature. We found that all 138 infants and children had a patent airway during sedation with this low-dose propofol-based regimen. The narrowest part of the pharyngeal airway was measured at the level of the base of the tongue. Anteroposterior dimensions were narrower than transverse dimensions in all age groups at all measurement sites. An additional finding was that transverse dimensions increased with age at all measurement sites, while anteroposterior dimensions did not increase comparably. All dimensions at all measurement sites were at least as wide than previously described during propofol sedation with comparable dosages (10,11). All MRI examinations could be completed successfully.

The narrowest part of the upper airway was measured at the base of the tongue. This finding concurs with the traditional view of several studies in adults, that airway obstruction may be caused predominantly by apposition of the base of the tongue to the posterior pharyngeal wall $(16,17)$, and challenges the results of more recent studies in children $(10,11)$. These studies concluded that anesthetic-induced attenuation of upper airway muscle activity results in an airway caliber decrease most pronounced at the level of the soft palate and the epiglottis. However, in these studies sample size was small and propofol dosage up to three times as high as the dosages administered to our patients. Furthermore, these studies were designed to determine the effects of increasing depth of propofol anesthesia on airway size, and not to evaluate an appropriate low propofol dosage for pediatric MRI sedation. We excluded a posterior displacement of the tongue due to head position as the reason for our results, because all mentioned studies investigated their patients with the head in the same neutral position. It is known that chin lift and mouth closure widens the entire upper airway mostly between the tip of the epiglottis and the posterior pharyngeal wall (18). However, we made no attempt to optimize mouth closure and chin lift, but we confirm the observation of a previous 
study (19), that the majority of children under propofol sedation maintain their jaw in the neutral position between opened and closed. Additionally, the inspiratory narrowing of the pharyngeal airway could have influenced our results. The only attempt to minimize this limitation was made by averaging the values of two subsequent slices at each measurement site. Image acquisition was not triggered manually to early expiration, because the relatively high respiratory rate of the investigated infants prevented us from acquiring images at a fixed point of respiratory cycle.

The finding that anteroposterior dimensions were narrower than transverse dimensions reflects previously described effects of propofol on pharyngeal airway morphology. Some authors suggest that propofol is associated with a differential effect between separate pharyngeal dilatator muscles, which is represented by changes of the upper airway shape during sedation and awakening from sedation $(19,20)$. Under their sedation regimen, the upper airway was oblong shaped, with the anteroposterior dimension larger than the transverse dimension. On awakening, the transverse dimension was larger than the anteroposterior dimension, whereas the CSA was unchanged (12). The authors administered $200 \mathrm{mcg} \cdot \mathrm{kg}^{-1} \cdot \mathrm{h}^{-1}$ propofol, which is more than twice the dosage our patients received. Therefore we suggest that airway shape in our patients better represents the morphology during the awake state, which emphasizes the safety of our sedation regimen.

The finding that transverse dimensions increased with age while anteroposterior dimensions did not increase comparably, has not been described previously. Data of pharyngeal airway measurements in a comparable large collective are missing, and only one study determined developmental changes of the laryngeal dimensions in sedated children (19). However, measurements were performed more caudad between the vocal cords and the cricoid ring, and the authors concluded that anteroposterior and transverse dimensions increased linearly with age. If we assume that the more cephalad airway may have the same relation between anteroposterior and transverse dimension, we attribute the minor anteroposterior increase of 
our patients to an additional effect of propofol sedation on the pharyngeal airway. Increasing depth of propofol anesthesia seems to decrease airway caliber predominantly to anteroposterior narrowing (10).

One may argue that adding the partial agonist-antagonist opioid nalbuphine to a sedation regimen is not necessary for the inherently painless MRI examination. According to recent guidelines, sedatives or hypnotics alone have to be preferred for non-painful procedures (13). However, nalbuphine was included in our protocol, since nalbuphine $0.1 \mathrm{mg} \cdot \mathrm{kg}^{-1}$, administered before anesthesia induction, is described to decrease the frequency of spontaneous, unintentional movements induced by propofol (21). Furthermore, previous publications demonstrate that nalbuphine, administered at the end of MRI examinations, prevents emergence agitation (22). Due to the assumed additional sedative effect of nalbuphine to propofol, we were able to complete all MRI examinations without interruption with one of the lowest propofol doses (9). Pure propofol regimen seem to require doses up to $200 \mathrm{mcg} \cdot \mathrm{kg}^{-1} \cdot \mathrm{h}^{-1}$, and even then the rate of MRI pausing due to unintentional movements is $10-20 \%(4,23)$.

In summary, we demonstrated with objective measurements of the airway morphology via MRI in a large collective of infants and children with normal upper airway anatomy and neither obesity nor extreme tonsillar hypertrophy that our low-dose propofol-based sedation is safe regarding airway patency. 


\section{Legends}

Figure 1

A 3D gradient echo T1-weighted sequence in axial/oblique plane at the level of the soft palate. The arrow indicates the anteroposterior dimension.

Figure 2

A 3D gradient echo T1-weighted sequence in axial/oblique plane at the level of the base of the tongue. The arrow indicates the transverse dimension.

Figure 3

A 3D gradient echo T1-weighted sequence in axial/oblique plane at the level of the tip of the epiglottis. The arrow points at the measurement of the cross-sectional area.

Figure 4

Correlations of airway dimensions with age (Pearson's r). 


\section{References}

1 Mallory MD, Baxter AL, Kost SI. Propofol vs pentobarbital for sedation of children undergoing magnetic resonance imaging: results from the Pediatric Sedation Research Consortium. Paediatr Anaesth 2009; 19: 601-611.

2 Eastwood PR, Platt PR, Shepherd K, Maddison K, Hillman DR. Collapsibility of the upper airway at different concentrations of propofol anesthesia. Anesthesiology 2005; 103: $470-477$.

3 Mahmoud M, Radhakrishman R, Gunter J, et al. Effect of increasing depth of dexmedetomidine anesthesia on upper airway morphology in children. Paediatr Anaesth 2010; 20:506-15.

4 Usher AG, Kearney RA, Tsui BC. Propofol total intravenous anesthesia for MRI in children. Paediatr Anaesth 2005; 15: 23-28.

5 Levati A, Colombo N, Arosio EM, et al. Propofol anaesthesia in spontaneously breathing paediatric patients during magnetic resonance imaging. Acta Anaesthesiol Scand 1996; 40: 561-565.

6 Kol IO, Egilmez H, Kaygusuz K, Gursoy S, Mimaroglu C. Open-label, prospective, randomized comparison of propofol and sevoflurane for laryngeal mask anesthesia for magnetic resonance imaging in pediatric patients. Clin Ther 2008; 30: 175-181.

7 Bordes M, Semjen F, Sautereau A, et al. [Which anaesthesia for children undergoing MRI? An Internet survey in the French university hospitals]. Ann Fr Anesth Reanim 2007; 26: 287-291.

8 Young AE, Brown PN, Zorab JS. Anaesthesia for children and infants undergoing magnetic resonance imaging: a prospective study. Eur J Anaesthesiol 1996; 13: 400403. 
9 Machata AM, Willschke H, Kabon B, Kettner SC, Marhofer P. Propofol-based sedation regimen for infants and children undergoing ambulatory magnetic resonance imaging. Br J Anaesth 2008; 101: 239-243.

10 Crawford MW, Rohan D, Macgowan CK, Yoo SJ, Macpherson BA. Effect of propofol anesthesia and continuous positive airway pressure on upper airway size and configuration in infants. Anesthesiology 2006; 105: 45-50.

11 Evans RG, Crawford MW, Noseworthy MD, Yoo SJ. Effect of increasing depth of propofol anesthesia on upper airway configuration in children. Anesthesiology 2003; 99: 596-602.

12 Litman RS, Weissend EE, Shrier DA, Ward DS. Morphologic changes in the upper airway of children during awakening from propofol administration. Anesthesiology 2002; 96: 607-611.

13 Cote CJ, Wilson S. Guidelines for monitoring and management of pediatric patients during and after sedation for diagnostic and therapeutic procedures: an update. Pediatrics 2006; 118: 2587-2602.

14 Practice guidelines for sedation and analgesia by non-anesthesiologists. Anesthesiology 2002; 96: 1004-1017.

15 Malviya S, Voepel-Lewis T, Tait AR, Merkel S, Tremper K, Naughton N. Depth of sedation in children undergoing computed tomography: validity and reliability of the University of Michigan Sedation Scale (UMSS). Br J Anaesth 2002; 88: 241-245.

16 Fink BR. Roentgenographic studies of the oropharyngeal airway. Anesthesiology 1957; 18: 711-718.

17 Morikawa S, Safar P, Decarlo J. Influence of the headjaw position upon upper airway patency. Anesthesiology 1961; 22: 265-270.

18 Reber A, Wetzel SG, Schnabel K, Bongartz G, Frei FJ. Effect of combined mouth closure and chin lift on upper airway dimensions during routine magnetic resonance 
imaging in pediatric patients sedated with propofol. Anesthesiology 1999; 90: 16171623.

19 Litman RS, Weissend EE, Shibata D, Westesson PL. Developmental changes of laryngeal dimensions in unparalyzed, sedated children. Anesthesiology 2003; 98: 41-45.

20 Isono S. Developmental changes of pharyngeal airway patency: implications for pediatric anesthesia. Paediatr Anaesth 2006; 16: 109-122.

21 Borgeat A, Fuchs T, Wilder-Smith O, Tassonyi E. The effect of nalbuphine and droperidol on spontaneous movements during induction of anesthesia with propofol in children. J Clin Anesth 1993; 5: 12-15.

22 Dalens BJ, Pinard AM, Letourneau DR, Albert NT, Truchon RJ. Prevention of emergence agitation after sevoflurane anesthesia for pediatric cerebral magnetic resonance imaging by small doses of ketamine or nalbuphine administered just before discontinuing anesthesia. Anesth Analg 2006; 102: 1056-1061.

23 Bryan YF, Hoke LK, Taghon TA, et al. A randomized trial comparing sevoflurane and propofol in children undergoing MRI scans. Paediatr Anaesth 2009; 19: 672-681. 
Table 1: Demographic and morphometric data

\begin{tabular}{|c|c|c|c|c|}
\hline Age (month) & $<12$ & $12-<36$ & $36-<72$ & $P$ \\
\hline Number of patients & 27 & 40 & 71 & \\
\hline Age (month) & $6(3,10)$ & $21(17,27)$ & $48(42,58)$ & \\
\hline Weight (kg) & $6.9 \pm 3$ & $11.1 \pm 2$ & $17.7 \pm 4.1$ & $<0.001$ \\
\hline Height (cm) & $64(54,73)$ & $86(80,91)$ & $104(100,108)$ & $<0.001$ \\
\hline CSA sp $\left(\mathrm{mm}^{2}\right)$ & $103(84,127)^{* \#}$ & $135(103,184)$ & $167(119,196)$ & $<0.001$ \\
\hline AP sp (mm) & $8.2(7.6,9.4)^{\#}$ & $9.2(7.8,11.1)$ & $10.1(8.7,11.5)$ & 0.002 \\
\hline T sp (mm) & $13(11.6,14.5)^{* \#}$ & $16.5(13.8,18.5)$ & $17.7(15.6,19.4)$ & $<0.001$ \\
\hline $\operatorname{CSA~t~}\left(\mathrm{mm}^{2}\right)$ & $79(66,101)^{* \#}$ & $120(101,153)$ & $129(94,165)$ & $<0.001$ \\
\hline AP t (mm) & $7.3(6.1,8.2)^{* \#}$ & $9.1(7.7,10.4)$ & $9(8,10.8)$ & $<0.001$ \\
\hline $\mathrm{T} \mathrm{t}(\mathrm{mm})$ & $12.4(10.6,14) *$ & $15.1(12.8,17.5)$ & $15.8(13.1,18.9)$ & $<0.001$ \\
\hline CSA e $\left(\mathrm{mm}^{2}\right)$ & $89(78,127)^{* \# \#}$ & $137(113.1,170)$ & $143(111,175)$ & $<0.001$ \\
\hline AP e $(\mathrm{mm})$ & $8.6(6.5,9.9)^{*}$ & $9.9(8.6,11.5)$ & $9.3(7.9,11.3)$ & 0.048 \\
\hline $\mathrm{Te}(\mathrm{mm})$ & $13.2(11.5,14.5)^{* \#}$ & $16.5(14.9,18.2)^{\&}$ & $18.2(16.2,20)$ & $<0.001$ \\
\hline
\end{tabular}

$*<12$ significantly different to $12-<36$

$\#<12$ significantly different to $36-<72$

\& 12- $<36$ significantly different to $36-<72$

Data are presented as means $\pm \mathrm{SD}$, or median (IQR)

Comparisons between the age groups were performed with Kruskal-Wallis test. Post hoc comparisons were performed using Bonferroni corrections. 
Abbreviations: CSA sp - cross-sectional area at the level of the soft palate, AP sp anteroposterior dimension at the level of the soft palate, $\mathrm{T} s \mathrm{sp}-$ transverse dimension at the level of the soft palate, CSA t - cross-sectional area at the level of the base of the tongue, AP t - anteroposterior dimension at the level of the base of the tongue, $\mathrm{T} \mathrm{t}$ - transverse dimension at the level of the base of the tongue, CSA e - cross-sectional area at the level of the tip of the epiglottis, AP e - anteroposterior dimension at the level of the tip of the epiglottis, $\mathrm{T} \mathrm{e}-$ transverse dimension at the level of the tip of the epiglottis; 


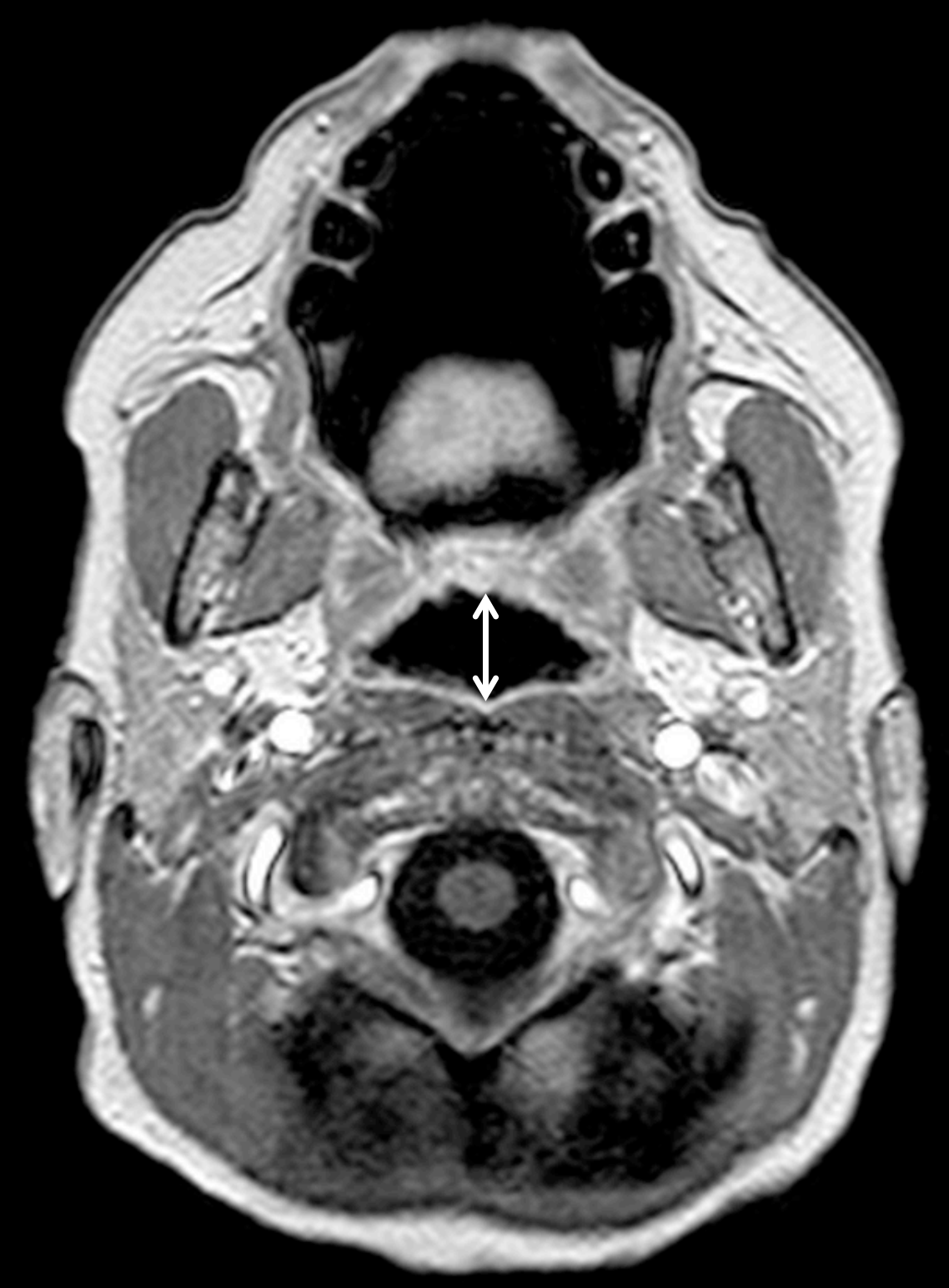




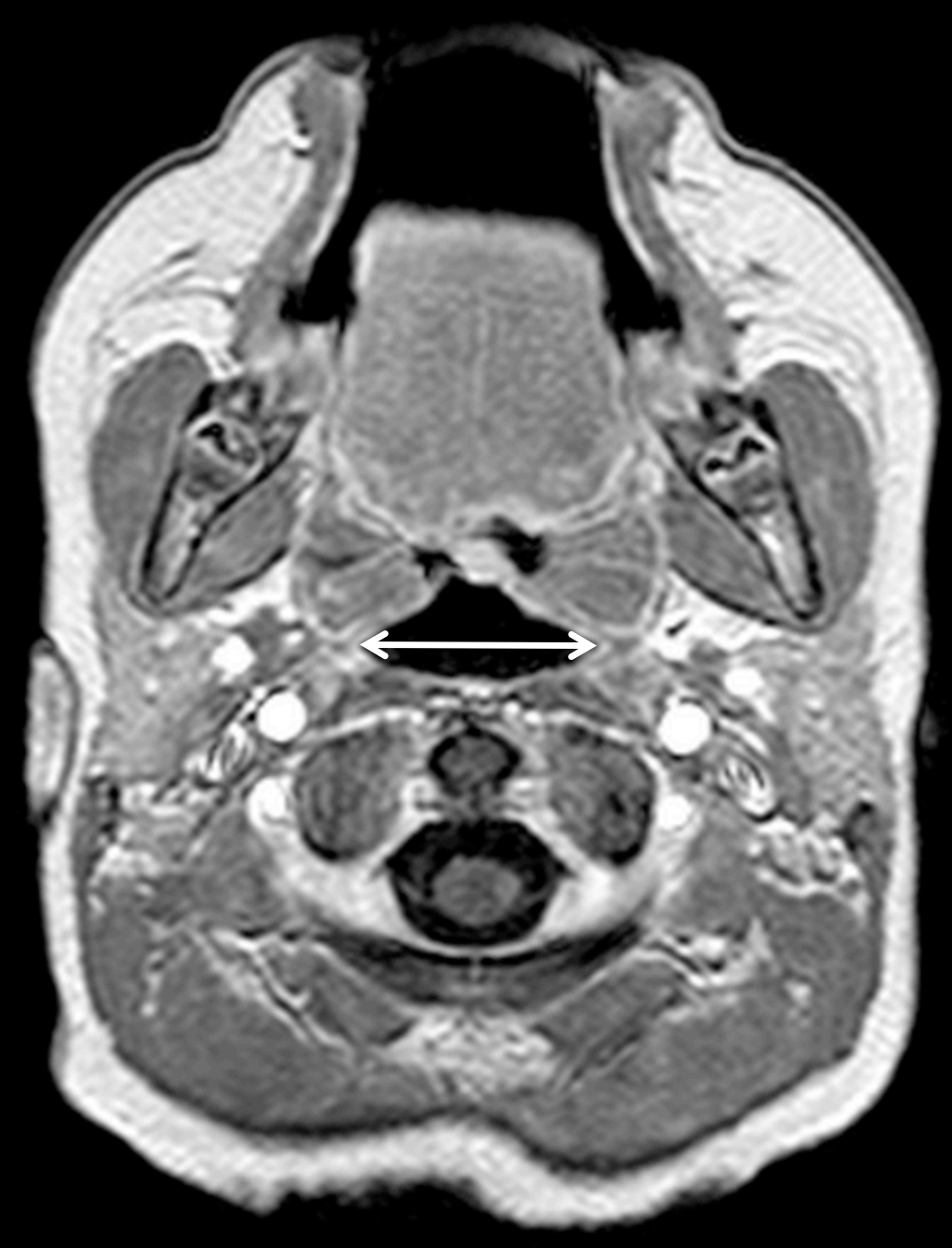




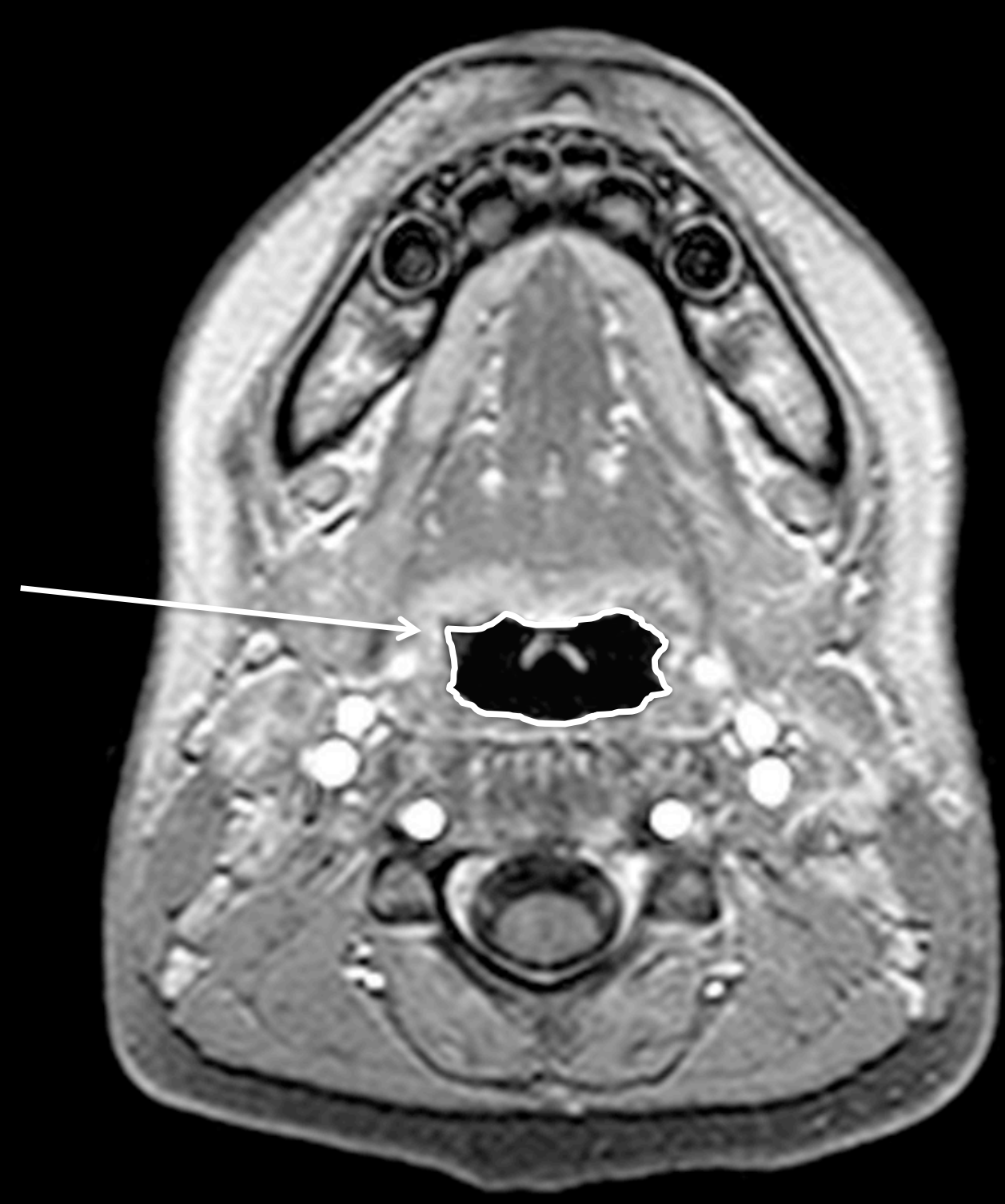




\section{AP dimension Transverse dimension CSA}

Soft palate
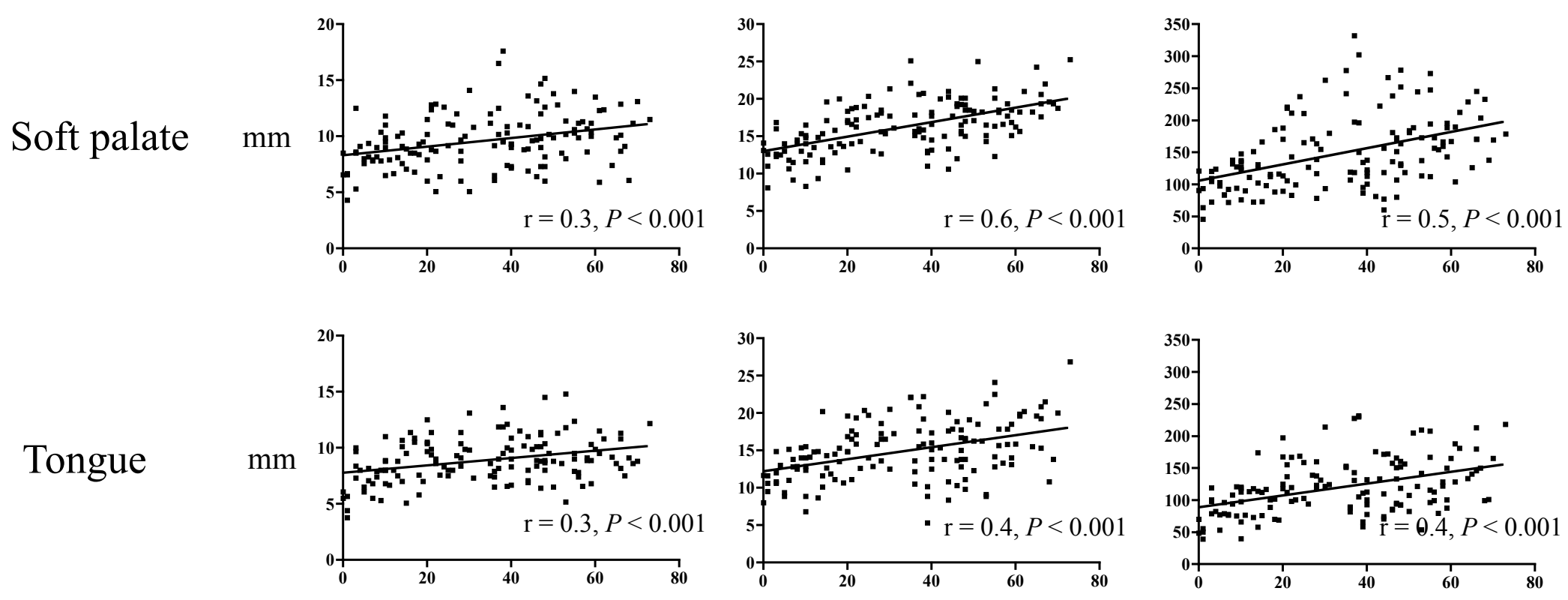

Epiglottis
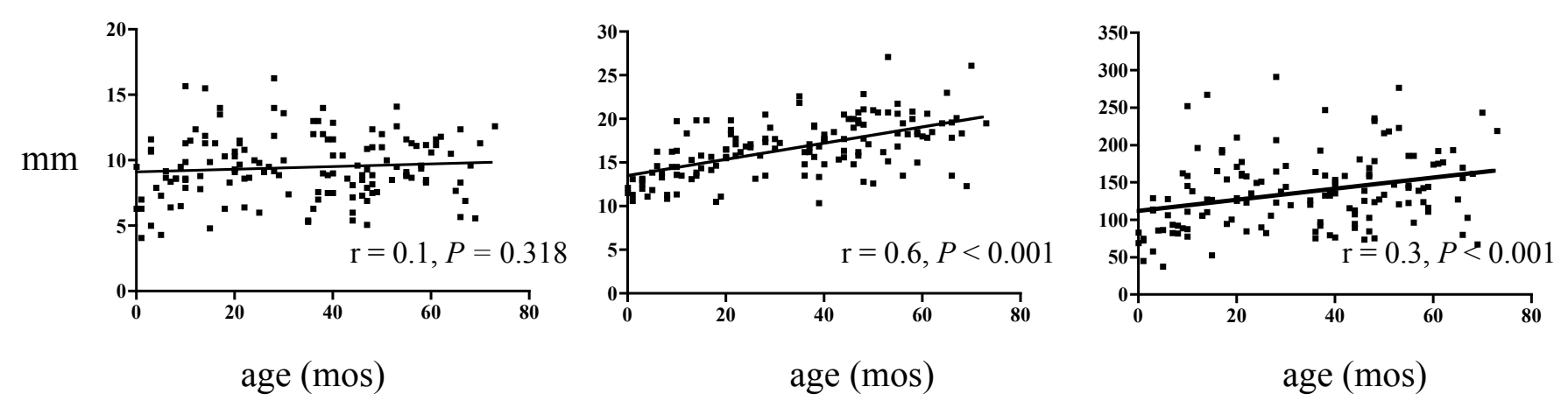